\title{
Bladder Urothelial Carcinoma with Peritoneal Involvement: Benefit of Continuous Maintenance Chemotherapy
}

\author{
CHUANG-CHI LIAW ${ }^{1}$, CHENG-KENG CHUANG ${ }^{2}$, YING-HSU CHANG ${ }^{2}$, TZU-YAO LIAO ${ }^{1}$, \\ JOHN WEN-CHENG CHANG ${ }^{1}$ and YU-HSIANG JUAN ${ }^{3}$ \\ ${ }^{1}$ Division of Hemato-Oncology, Department of Internal Medicine, \\ ${ }^{2}$ Department of Urology, and ${ }^{3}$ Department of Radiation, \\ Chang-Gung Memorial Hospital and Chang-Gung University College of Medicine, Taoyuan, Taiwan, R.O.C.
}

\begin{abstract}
Aim: We investigated bladder urothelial carcinoma with peritoneal involvement. Patients and Methods: Inclusion criteria were: pathology-confirmed urothelial carcinoma; peritoneal spread identified on computed tomographic (CT) scans performed initially or after either cystectomy or concomitant chemoradiotherapy (CCRT), and absence of visceral metastases; and chemotherapy administered after peritoneal spread was diagnosed. Results: Forty-seven cases included initial modes of therapy with chemotherapy in 24 patients (51\%), cystectomy in 17 (36\%), and CCRT in six (13\%), only given as a result of under-staging. After local therapy, these patients received a continuous maintenance chemotherapy regimen of 5-fluorouracil, leucovorin, cisplatin, and gemcitabine. The overall response rate was $85 \%$, and the side-effects were mild and tolerated. The median survival time was 28 months. The survival time of cases initially treated only with chemotherapy was not statistically different to that of those with local disease. Conclusion: Bladder urothelial carcinomas with peritoneal involvement can benefit from continuous maintenance chemotherapy.
\end{abstract}

The bladder is a sub-peritoneal organ, and the peritoneum covers its posterosuperior surface. Along its lateral margins, the peritoneum attaches to the lateral walls of the pelvis, and the anterior, lateral, and lower parts of the posterior surface of the bladder are not connected to the peritoneum. The peritoneum consists of two layers: the

Correspondence to: Chuang-Chi Liaw, MD, Division of HematoOncology, Department of Internal Medicine, Chang-Gung Memorial Hospital, 5, Fusing St., Gueishan Township, Taoyuan County, 333, Taiwan, R.O.C. Tel: +88633281200 ext 8825, Fax: +8863278211 , e-mail: e102309@adm.cgmh.org.tw

Key Words: Bladder urothelial carcinoma, peritoneal metastases, computed tomography, cystectomy, chemotherapy. visceral and the parietal peritoneum. The parietal peritoneum is attached to the abdominal and pelvic walls (1). The pelvic peritoneal space is defined by the inferior reflection of the peritoneum over the fundus of the urinary bladder and the front of the rectum at the junction of the middle and lower thirds of the rectum (1). When disease spreads from the subperitoneal space to the peritoneal cavity via the peritoneal lining, transperitoneal spread can occur. According to the seventh edition of the American Joint Committee on Cancer (AJCC) TNM system, stage T4b bladder cancer describes a tumor that has spread to the pelvic or abdominal wall (2). Tumors can enter the abdominal wall from the bladder dome, and the peritoneum (3) and can invade the pelvic sidewall from the posterolateral bladder wall or involve the peritoneal reflexion $(4,5)$. Spread of bladder urothelial carcinoma to the peritoneum is frequently encountered (4-8).

Computed tomographic (CT) scans are useful for determining the stage and extent of bladder cancer, and can also detect peritoneal metastasis (6-9). However, identifying peritoneal spread before treatment to determine the best course of action is difficult. Black et al. reported that longterm survival can be achieved in some patients with cT4b bladder cancer undergoing chemotherapy and extirpative surgery (10). However, Liberman et al. mentioned that radical cystectomy of patients with pT4b disease had higher cancer-specific mortality (11).

In this case series study, we investigate the clinical course of bladder urothelial carcinoma with peritoneal involvement, and discuss diagnosis and management, especially chemotherapy.

\section{Patients and Methods}

Study population. This retrospective case series study was conducted using data collected from patients with bladder cancer who were admitted to the Oncology wards of Chang-Gung Memorial Hospital, Taoyuan, Taiwan, between January 2010 and December 2016. The majority of patients had urothelial carcinomas, and a medical oncologist specializing in urological cancer provided most of the 
data. The criteria for inclusion were: pathology-confirmed diagnosis of urothelial carcinoma; peritoneal spread identified by CT scans performed initially or after either radical/partial cystectomy or concomitant chemoradiotherapy (CCRT), and an absence of metastases in the visceral organs; and chemotherapy was administered after the diagnosis of peritoneal spread.

Of the 206 patients with bladder cancer assessed, 47 (23\%) met the criteria for inclusion in this study. Initial treatment included chemotherapy, radical or partial cystectomy, or CCRT. The bladder cancer stage was determined from CT scans $(12,13)$ according to the seventh edition of the AJCC TNM system (2), and the treatment response assessment for bladder cancer was based on the RECIST criteria (14).

Chemotherapy regimens included 5-fluorouracil, leucovorin, cisplatin, and gemcitabine (Gemmis; TTY, Taipei, Taiwan, ROC). The original dosage was $50 \mathrm{mg} / \mathrm{m}^{2}$ cisplatin by intravenous infusion for 3 hours on day 1 , plus $1,000 \mathrm{mg} / \mathrm{m}^{2}$ gemcitabine intravenous infusion for 30 minutes on days 1,15 and 22 in combination with a continuous intravenous infusion of $1000 \mathrm{mg} / \mathrm{m}^{2} 5$-fluorouracil and $100 \mathrm{mg}$ leucovorin for $46 \mathrm{~h}$ every 28 days. When renal function impairment was observed (serum creatinine value $>2 \mathrm{mg} / \mathrm{dl}$ ), cisplatin was changed to $150 \mathrm{mg} / \mathrm{m}^{2}$ carboplatin intravenous infusion for 2 hours. The schedule was modified during the chemotherapy course in the case of challenging admissions. Generally, we administered an inpatient chemotherapy regimen of 5-fluorouracil and leucovorin plus cisplatin with or without the gemcitabine regimen every 4 weeks, and gemcitabine was administered in the Outpatient Department every 2 weeks. Treatment strategies were initially aggressive, but were slowed down based on stable or improving condition and the results of imaging studies. We lengthened the chemotherapy interval or administered gemcitabine only if the patient was too old, had a severe comorbidity, or a poor performance status. Continuous maintenance chemotherapy was administered until tumor progression was detected, or the patient or doctor decided to discontinue therapy. Follow-up CT scans were performed every 3 months to assess tumor response.

Schedule of initial CCRT with combined radiotherapy and chemotherapy. The radiotherapy dose administered was 44-45 Gy in 22-26 fractions delivered to the whole pelvis, followed by a booster to the bladder, to a total dose of 60-61.2 Gy. The chemotherapy regimen was 5-fluorouracil, leucovorin, and cisplatin: $50 \mathrm{mg} / \mathrm{m}^{2}$ cisplatin intravenous infusion for 3 hours on day 1 , combined with a continuous intravenous infusion of $1,500 \mathrm{mg} / \mathrm{m}^{2}$ 5-fluorouracil and $150 \mathrm{mg}$ leucovorin for 46 hours every 28 days. The post-cystectomy CCRT radiotherapy dose was 45 Gy in 25 fractions to the pelvis, then a booster to the bladder fossa, up to a total dose of $54 \mathrm{~Gy}$, in 30 fractions.

Identification of inflammatory cytokines in patients with bladder cancer is necessary (15) due to paraneoplastic syndromes related to cytokine production, including thromboembolism complications, cachexia, and neoplastic fever, during the disease course $(16,17)$. Enoxaparin (Sanofi-Aventis) was administered to treat thromboembolism complications, medroxyprogesterone acetate for cancer cachexia, and naproxen for neoplastic fever.

Statistical methods. Continuous data (presented as the mean \pm standard deviation) were used to determine the number of chemotherapy cycles delivered by inpatient and outpatient services. The survival time was calculated from the start of therapy to death,
Table I. Characteristics of the 47 patients with bladder cancer with peritoneal involvement.

\begin{tabular}{lc}
\hline Characteristic & Value \\
\hline Median age (range), years & $67(40-85)$ \\
Gender & \\
$\quad$ Male/female & $34 / 13$ \\
Tumor extent, no./total no. (\%) & \\
Iliac lymph nodes* & $19 / 47(40)$ \\
Abdominal wall & $8 / 47(17)$ \\
Pelvic sidewalls & $3 / 47(6)$ \\
Prostate, uterus, or rectum & $6 / 47(16)$ \\
Entire bladder wall & $5 / 47(11)$ \\
Performance status, no./total no. $(\%)$ & \\
$0-1$ & $44 / 47(94)$ \\
$\geq 2$ & $3 / 47(6)$ \\
Initial understaging (<T4), no./total no. $(\%)$ & \\
Yes & $24 / 47(51)$ \\
No & $23 / 47(49)$ \\
Initial mode of therapy, no./total no. $(\%)$ & \\
Chemotherapy & $24 / 47(51)$ \\
Surgery & $17 / 47(36)$ \\
CCRT & $6 / 47(13)$ \\
\hline
\end{tabular}

CCRT: Concomitant chemoradiotherapy. *Two cases with tumors extending to the para-aortic lymph nodes; two cases with tumors extending to the para-aortic and supraclavicular lymph nodes.

Table II. Treatment response rate following chemotherapy and paraneoplastic syndromes reported.

\begin{tabular}{lc}
\hline & No. of patients $(\%)$ \\
\hline Response & \\
Overall response & $46 / 47(85)$ \\
Complete response & $2 / 47(4)$ \\
Partial response & $44 / 47(81)$ \\
Stable disease & $2 / 47(4)$ \\
Progressive disease & $6 / 47(11)$ \\
Paraneoplastic syndrome & $25 / 47(53)$ \\
Cachexia & $24 / 47(51)$ \\
Thromboembolic complications & $22 / 47(47)$ \\
Neoplastic fever & $3 / 47(6)$ \\
\hline
\end{tabular}

and the survival curves were determined using the Kaplan-Meier method. Significant differences between survival curves were measured using the log-rank test.

\section{Results}

Forty-seven consecutive bladder cancer patients met the inclusion criteria for this study, including 34 men and 13 women (median age $=67$ years; range $=40-85$ years). The patients' clinical characteristics are shown in Table I. All patients had peritoneal involvement, and a range of tumor 
A

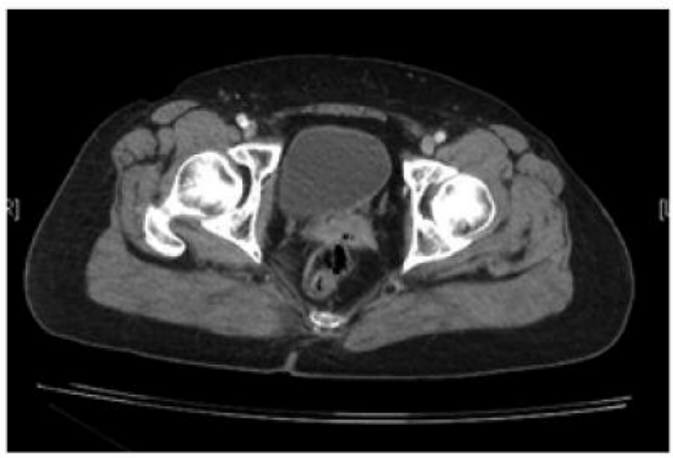

C

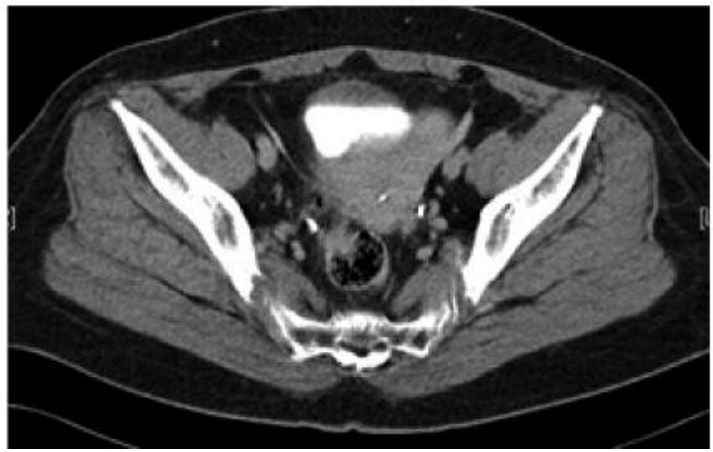

B

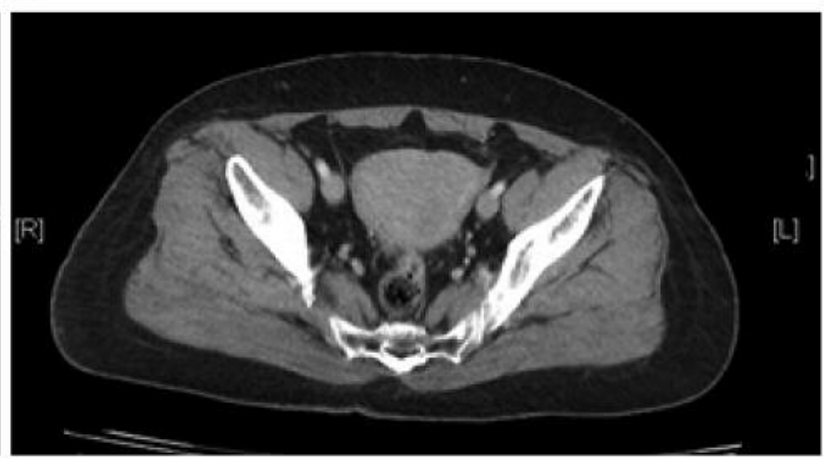

D

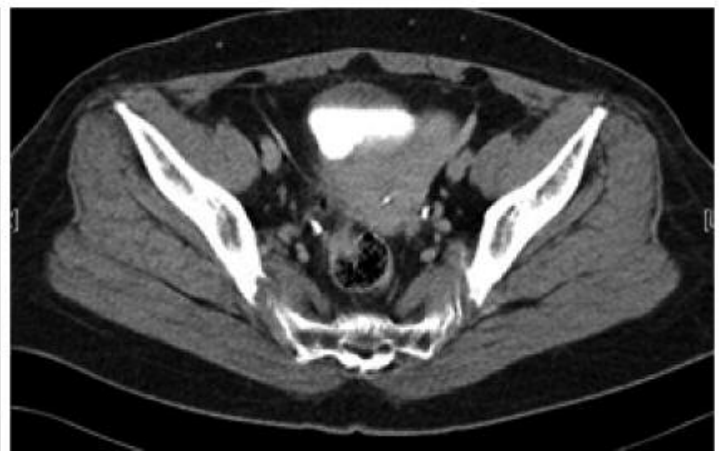

Figure 1. A 60-year-old woman with bladder urothelial carcinoma with peritoneal spread. The initial clinical stage was T4N1M1. She underwent continuous maintenance chemotherapy for four years. A and B: Computed tomographic scans showing tumor spread from the left posterolateral aspect of the bladder to the peritoneum. $C$ and D: Computed tomographic scans after she had received continuous maintenance chemotherapy showed tumor regression.

extents was represented: 19 patients $(40 \%)$ had a tumor involving iliac lymph nodes, including two involving paraaortic lymph nodes and two involving the supraclavicular lymph nodes; eight (15\%) had superior and anterior invasion into the abdominal wall; three $(6 \%)$ had tumors extending to the pelvic sidewall; six (13\%) had posterior invasion of the prostate, uterus, or rectum; and five (11\%) had circumferential tumors that surrounded the entire bladder wall. Forty-three patients (94\%) had an Eastern Cooperative Oncology Group (ECOG) performance status of 0 or 1 . Two had simultaneous bladder and renal pelvis urothelial carcinomas, and three had undergone nephroureterectomy for a previous upper urinary tract urothelial carcinoma (two cases involving the renal pelvis and one involving the ureter). Two cases had prior renal transplantation. Initial understaging (less than T4) on CT scans occurred for 22 patients $(44 \%)$.

Initial modes of therapy were chemotherapy in 24 patients (51\%), radical or partial cystectomy in 17 (36\%), and CCRT in six (13\%). The clinical staging before surgery was $\mathrm{T} 1$ in two patients, T2 in twelve, T3 in two, and T4 in one. The clinical staging before CCRT was T2 in five patients, and T1 in one. Inpatient chemotherapy regimens included 5fluorouracil, leucovorin, and cisplatin, with or without gemcitabine every 4 weeks. Five cases experienced renal function insufficiency (serum creatinine level $>2.0 \mathrm{mg} / \mathrm{dl}$ ) three initially and two during chemotherapy - and were given carboplatin instead of cisplatin. The outpatient chemotherapy regimen was gemcitabine monotherapy every 2 weeks. The mean number of inpatient chemotherapy cycles was $10 \pm 6$ (range, 1-24 cycles) and the mean number outpatient chemotherapy cycles was $21 \pm 16$ (range, 0-83 cycles). After a period of inpatient and outpatient chemotherapy, 13 patients chose to rest. There were nine patients who did not agree to long-term chemotherapy, three stopped chemotherapy based on their doctors' advice, and one ceased chemotherapy due to comorbidity.

The overall chemotherapy response rate was $85 \%$, including complete response in $4 \%$, partial response in $81 \%$, stable disease in $4 \%$, and progressive disease in $11 \%$ 
A

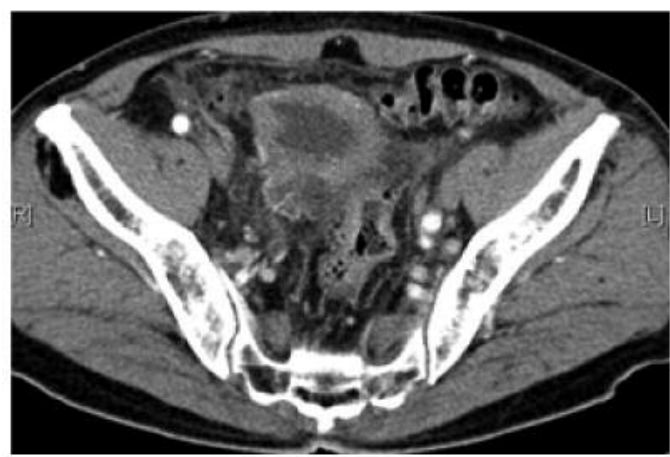

C

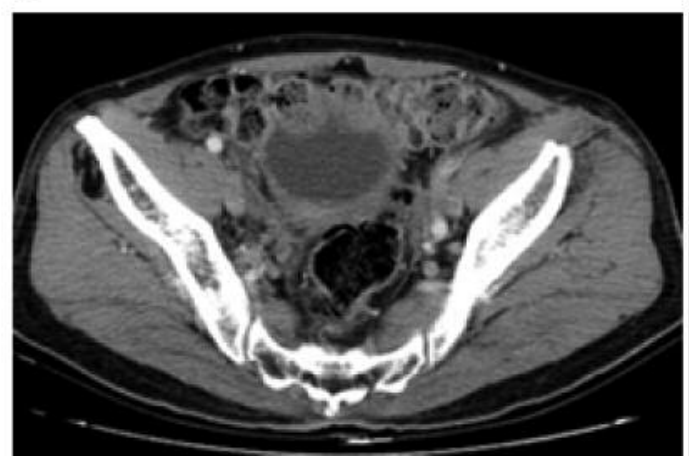

B

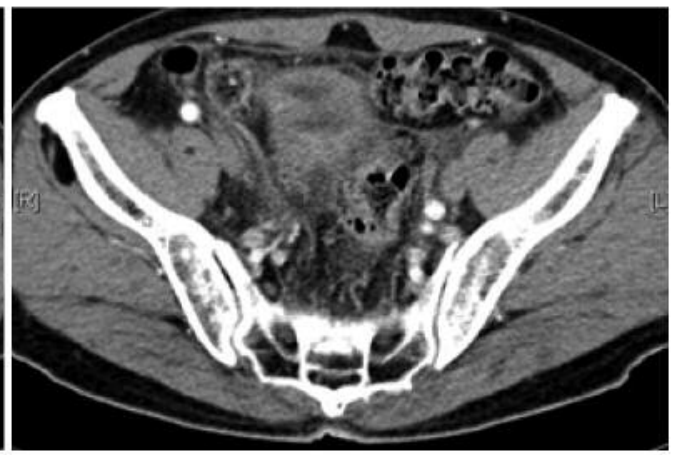

D

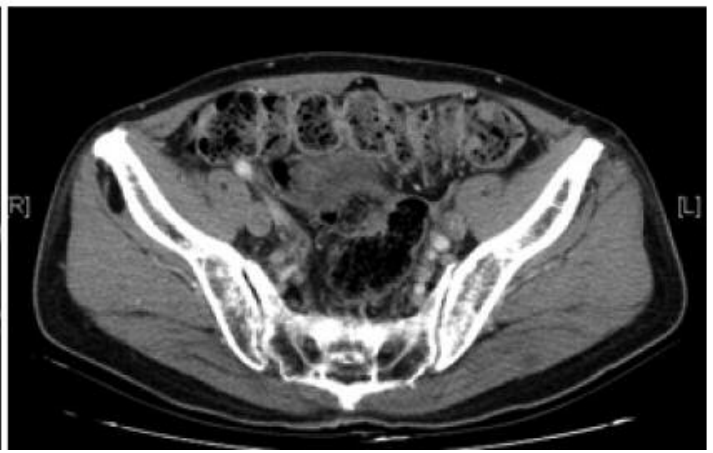

Figure 2. An 80-year-old man with bladder urothelial carcinoma with peritoneal spread. The initial clinical stage was T2NOM0. The patient underwent concomitant chemoradiotherapy (CCRT). Two months later, the tumor had progressed rapidly, with increased peritoneal lesions and lung metastases. A and B: Computed tomographic scans showing tumors infiltrating the entire bladder wall and spreading from the bladder dome to the peritoneum. C and D: Computed tomographic scans showing tumor progression with increased peritoneal extension following CCRT.

(Table II). The response evaluation sites were the bladder tumors, lymph nodes, and peritoneal lesions; however, thick or dirty lesions over the peritoneum made it difficult to assess the level of treatment response. Figure 1 shows a bladder tumor that exhibited a good partial response after chemotherapy only.

The side-effects of the chemotherapy were mild and tolerated, and sepsis occurred in only one patient during chemotherapy. During the treatment course, 25 patients (53\%) developed paraneoplastic syndrome. Thromboembolic complications detected by imaging were iliofemoral venous obstruction in nine patients, inferior vein thrombosis in four cases, pulmonary vein obstruction in three, and cerebral thrombosis in two.

The overall response rate for the 24 patients in the initialchemotherapy-only group was $92 \%$, including complete response in $8 \%$, partial response in $83 \%$, stable disease in $4 \%$, and progressive disease in $4 \%$. The mean number of inpatient chemotherapy cycles was $10 \pm 5$ (range $=1-20$ cycles), and of outpatient chemotherapy cycles was $21 \pm 18$ (range $=0-83$ cycles). One patient underwent radical cystectomy after one cycle of inpatient and four cycles of outpatient chemotherapy. Six patients elected for chemotherapy to be tapered off after some time. One patient received 11 cycles of inpatient and 83 cycles of outpatient chemotherapy, which was then tapered based on the doctor's advice; unfortunately, locoregional tumor recurrence over the whole bladder region occurred 17 months later. Another patient with pathology-proven urothelial carcinoma experienced tumor recurrence after receiving 10 cycles of inpatient and eight cycles of outpatient chemotherapy, with a weaning-off interval of 12 months. One patient received radiotherapy following the cessation of chemotherapy, and the continuous maintenance chemotherapy of another, who had pathology-proven urothelial carcinoma, involved 20 cycles of inpatient and 42 cycles of outpatient chemotherapy.

Of the six patients who received CCRT, none exhibited a positive tumor response; in fact, three experienced tumor progression following CCRT (see Figure 2). Of the 17 patients who underwent cystectomy, 13 underwent radical 
A

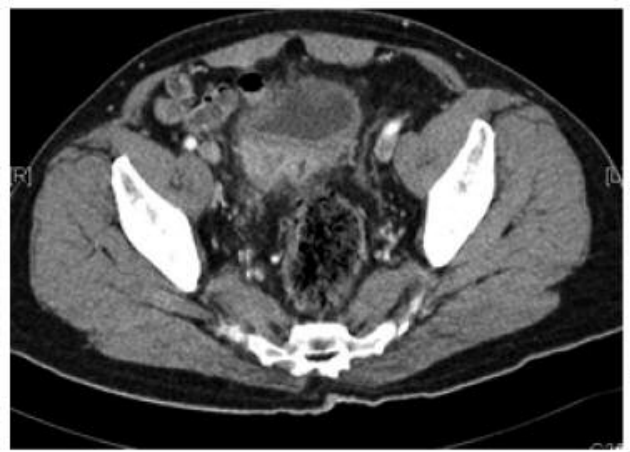

C

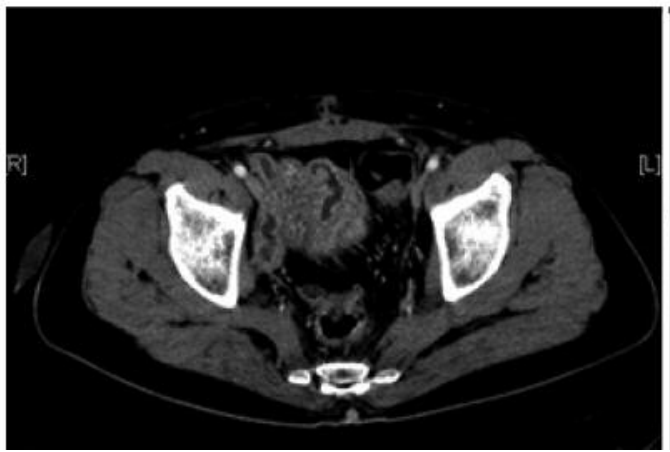

B

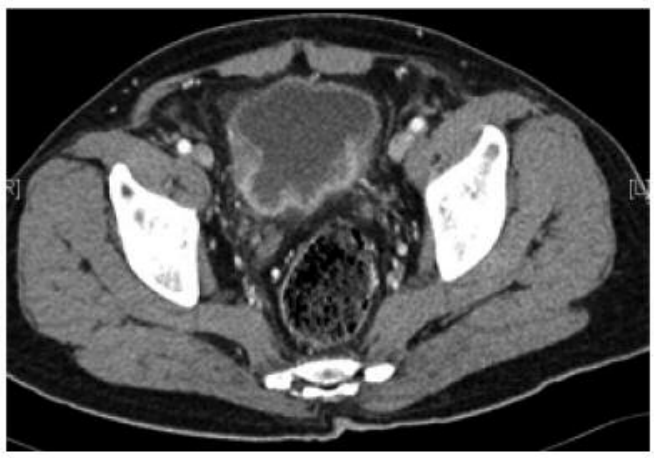

D

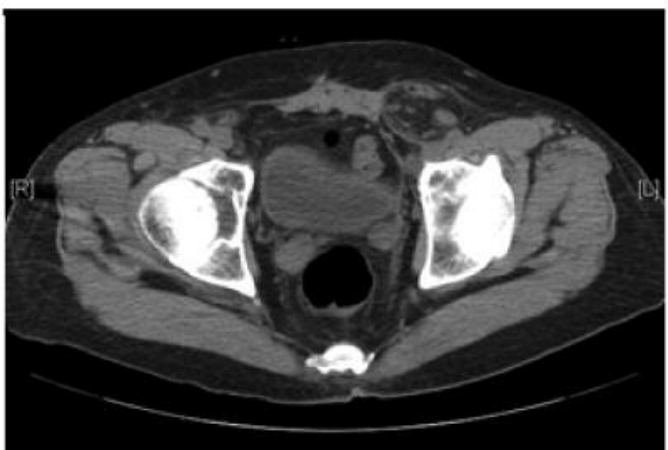

Figure 3. A 56-year-old man with bladder urothelial carcinoma with peritoneal spread. The initial clinical stage was T2NOMO. The patient underwent radical cystectomy and the pathological stage of the specimen was T3bNOMO. Postoperatively, the patient received concomitant chemoradiotherapy (CCRT) followed by continuous maintenance chemotherapy for 3.5 years. A and B: Computed tomographic scans showing tumors spreading from the right anterolateral bladder wall and dome to the peritoneum and the right abdominal wall. C: Computed tomographic scan showing right pelvic peritoneal thickening and abdominal wall invasion 3 months after surgery. D: Computed tomographic scan showing tumor regression after the patient had received CCRT and continuous maintenance chemotherapy.

cystectomy and four underwent partial cystectomy. Eight cases received postoperative therapy when the CT scan showed evidence of a recurrent peritoneal tumor or they became symptomatic; 14 received chemotherapy and three received CCRT. The mean duration of therapy following surgery was $4.4 \pm 2.8$ months (range $=1-10$ months). Figure 3 shows an example of tumor regression after CCRT and subsequent continuous maintenance chemotherapy following radical cystectomy. Nine cases, including eight chemotherapy and one CCRT. Figure 4 shows an example of tumor regression after chemotherapy only, following evidence of peritoneal tumor recurrence on CT scans after partial cystectomy. The partial response rate after chemotherapy for these 23 patients was $74 \%$, the stable disease rate was $4 \%$, and the progressive disease rate was $22 \%$. The mean number of inpatient chemotherapy cycles was $11 \pm 7$ (range $=2-24$ cycles) and the mean number of outpatient chemotherapy cycles was $20 \pm 14$ (range $=0-51$ cycles). Seven patients elected to rest. Three patients experienced an inguinal hernia (probably related to tumor invasion of the abdominal wall; Figure 4D), of whom two underwent herniorrhaphy but showed abdominal-wall regional tumor progression with peritoneal seeding following the procedure.

The follow-up period ranged from 3 to 80 months. Twenty-six patients were followed-up until their death; two were lost to follow-up. Nineteen patients were still alive at the time of writing. The median survival time was 28 months (range of 3 to $>80$ months). The median survival time was 26 months for the initial-chemotherapy-only group versus 32 months for the group treated with initialcystectomy or CCRT, According to a log-rank test $(p=0.51)$, the survival time of the two groups was not statistically different (Figure 5). Of the mortalities, death resulted from loss of consciousness in 25 cases and respiratory failure in one. 

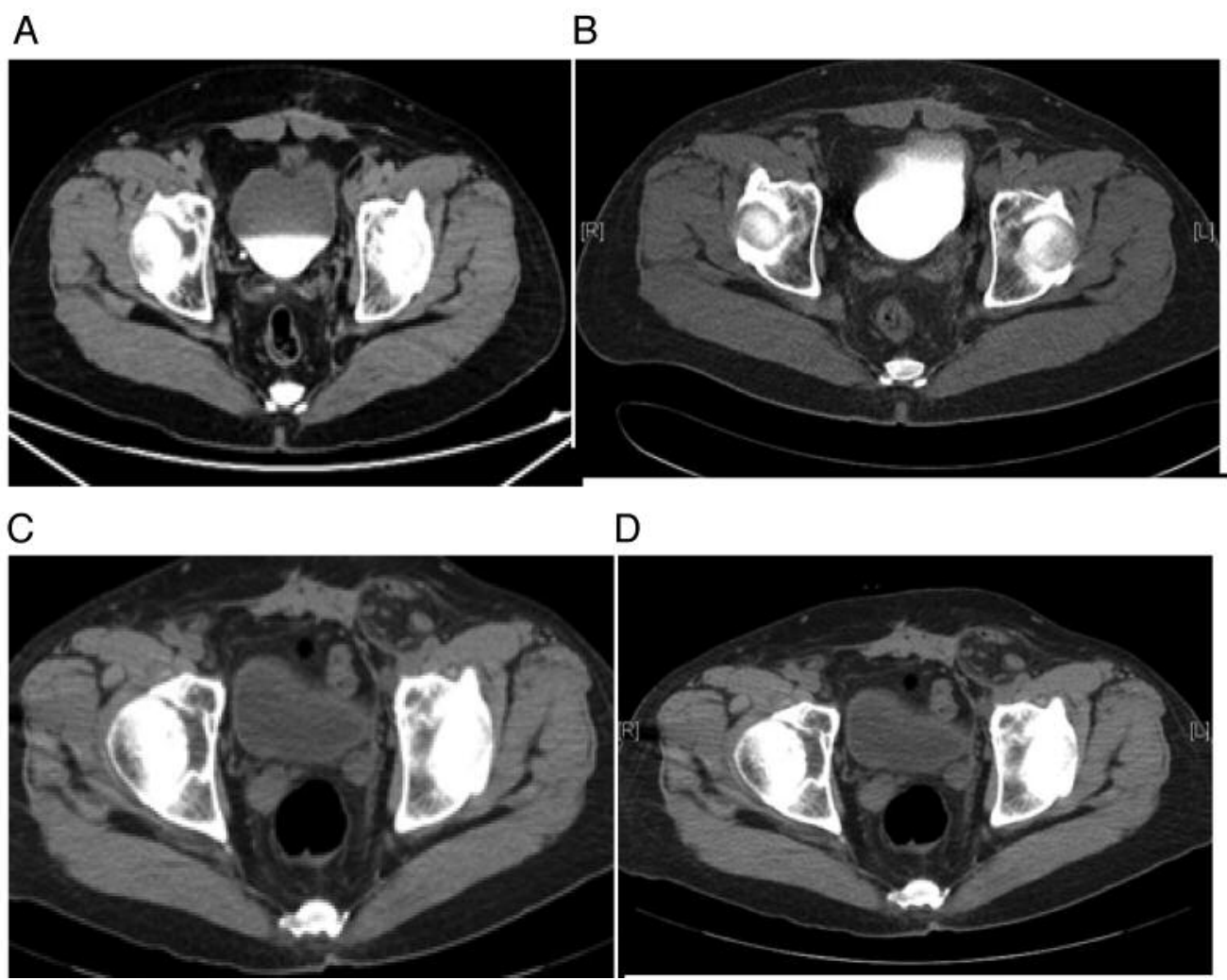

D

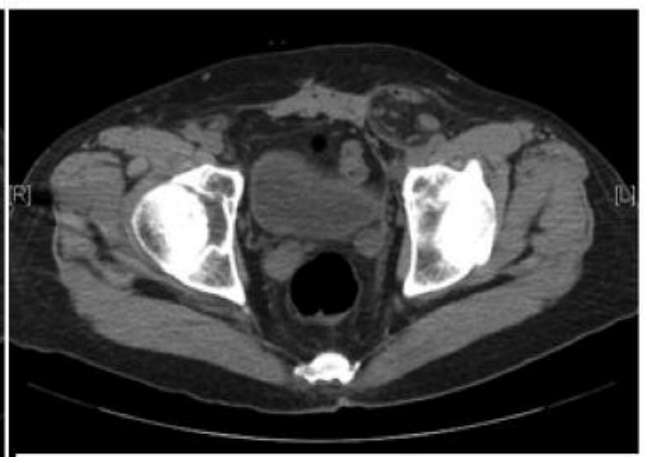

Figure 4. A 59-year-old man with bladder urothelial carcinoma with peritoneal spread. The initial clinical stage was T3NOMO. The patient underwent partial cystectomy; the pathological stage of the specimen was T1NOMO. Six months postoperatively, tumor recurrence was noted with peritoneum and left-abdominal-wall spread. The patient received continuous maintenance chemotherapy for three years. A and B: Computed tomographic scans showing show tumors extending from the anterior bladder wall to the peritoneum and the left abdominal wall. Computed tomographic scan showing tumor regression $(C)$ and the formation of an inguinal hernia $(D)$ after the patient had received continuous maintenance chemotherapy.

\section{Discussion}

CT scans play an important role in the diagnosis of peritoneal spread $(7,8)$, and understanding the anatomical relationship between the peritoneum and the bladder is crucial $(1,2)$. Tumors can progress to anterior invasion from the superior or the anterior side, from the bladder dome to the abdominal wall, can spread anterolaterally or posteriolaterally to the pelvic peritoneum, and can attach circumferentially to the peritoneum and surround the entire bladder wall. Forty percent of our patients had tumors that extended to the iliac lymph nodes. Peritoneal spread, iliac lymph node involvement, and T4b lesion staging based on the seventh edition of the AJCC TNM system were all classified as stage 4 disease.

The chemotherapy regimen used in our study included 5-fluorouracil, leucovorin, cisplatin, and gemcitabine. Combination chemotherapy with gemcitabine plus cisplatin,

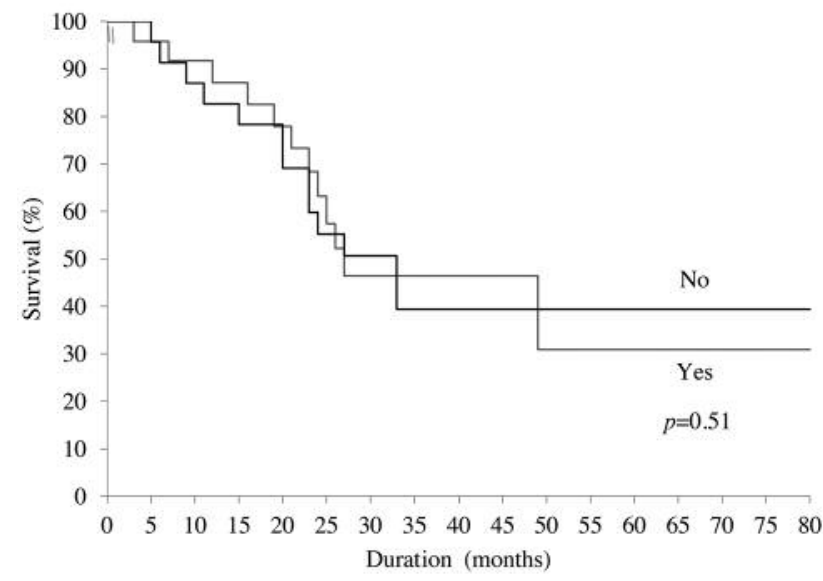

Figure 5. The survival time for the groups treated with initial chemotherapy only and those with initial cystectomy or concomitant chemoradiotherapy. According to the log-rank test, median survival $(28$ months versus 32 months) did not differ significantly between groups. 
or 5-fluorouracil and leucovorin plus cisplatin are both considered aggressive treatment for advanced or metastatic bladder urothelial carcinoma $(18,19)$. However, patients with bladder cancer are usually older and have renal function impairment with a post-unilateral or renal transplant status (20-22). Administration of $50 \mathrm{mg} / \mathrm{m}^{2}$ cisplatin every 4 weeks can prolong its use and reduce its toxicity (23) indeed, significantly improved survival of patients with metastatic colorectal cancer can be achieved using continuous maintenance chemotherapy $(24,25)$. Karogirou et al. reported that maintenance monotherapy with gemcitabine following primary cisplatin-based combination chemotherapy in patients surgically treated for advanced urothelial carcinoma significantly improved treatment outcomes (26). In our patients, the overall response rate was $85 \%$, and the median survival time was 28 months.

The disease status of the initial-chemotherapy-only patients usually involved obvious peritoneal lesions. Their overall treatment response rate was over $90 \%$, with a median survival time of 26 months. Only one patient underwent radical cystectomy after chemotherapy. We, therefore, support the use of neoadjuvant chemotherapy for muscleinvasive bladder cancer (27). However, a subset of patients receiving chemotherapy will still experience disease progression (28)

In this study, initial treatment with cystectomy or CCRT was related to understaging. Initial-cystectomy patients generally did not exhibit obvious peritoneal lesions. We also excluded patients with visceral metastases at the time of the diagnosis of peritoneal metastases. While immediate versus deferred chemotherapy after radical cystectomy in patients with pT3-pT4 or N+ M0 urothelial carcinoma of the bladder showed no difference in outcome (29), early initiation of chemotherapy following complete resection of advanced ovarian cancer has been shown to be associated with improved survival (30). Those of our patients who received initial CCRT showed no positive treatment response, and some even exhibited tumor progression. This is inconsistent with our previous study, which suggested that CCRT is a feasible and promising treatment for invasive bladder cancer (31). This is probably due to the presence of peritoneal lesions outside the radiation field. Patients treated with initial cystectomy or CCRT had an overall response rate of $74 \%$ with chemotherapy, however. The median survival time from initial cystectomy or CCRT was 32 months, and there was no significant difference in survival time between the groups treated with initial chemotherapy only and those treated with initial cystectomy or CCRT in our study.

The tumor microenvironment has been shown to affect tumor progression, and has been detected in bladder cancer tissues (32). Evidence suggests that critical elements of the tumor microenvironment are immune and inflammatory cells, blood and lymphatic vascular networks, fibroblasts, and the extracellular matrix (33). Cytokine production and alteration of hemostasis in cancer can promote tumor progression (16). Cancer cachexia, thromboembolic complications, and neoplastic fever are all linked to cytokine production by inflammatory cells (34-36), although thromboembolic complications are also associated with vascular disorders. Anti-cytokine therapy, such as progesterone acetate, nonsteroidal anti-inflammatory drugs, and low-molecular-weight heparin, can improve these complications, probably by modifying the tumor microenvironment to prevent tumor growth (37).

Cancer-associated fibroblasts (CAFs), a major component of the cancer stroma, have a prominent role in the progression, growth, and spread of cancers $(38,39)$, and can be involved in angiogenesis and the response to injury. CAFs are thought to be derived from mesothelial cells via mesothelial-to-mesenchymal transition in peritoneal metastasis (40). The extracellular matrix either represents a defense mechanism by non-malignant host cells against invading cancer cells, or promotes tumorigenesis. Formation of the extracellular matrix is essential for processes such as growth, wound healing, and fibrosis (41). In our patients, chemotherapy was able to reduce the peritoneal tumor burden and facilitate peritoneal wound healing in order to prevent tissue damage. A dirty and thickened peritoneum represented cancer cells in the peritoneum following chemotherapy; however, the number of cancer cells and their fibrotic tissue could not be determined. These phenomena are probably linked to the presence of fibroblasts, since fibrotic tissue can prevent tumor progression. Some patients were able to stop chemotherapy for a period of time and remain in a stable condition. Two of our patients who underwent herniorrhaphy to repair a hernia sac exhibited abdominal-wall and regional peritoneal progression after surgery. Although the true etiology of a dirty and thickened peritoneum is not known, CAFs and the extracellular matrix may have played an important role in our patients.

Our study has several important limitations. Firstly, the data were collected from a retrospective case cohort from a single center, mainly from one physician. Secondly, the inpatient and out-patient chemotherapy duration was not uniform. Thirdly, the diagnoses of peritoneal involvement and further investigations lacked histological confirmation.

\section{Conclusion}

Bladder urothelial carcinomas with peritoneal involvement can benefit from chemotherapy. Continuous maintenance chemotherapy can prolong patient survival for more than 2 years. Pre-therapy staging based on CT scan is important, especially for detecting tumors with peritoneal involvement. Understanding the concept of the tumor microenvironmement can help to treat these patients. 


\section{Ethics Statement}

The Authors declare that an Institutional Review Board/Ethics Committee determined that patient informed consent was not required. The study was approved by the Chang Gung Medical FoundationInstitutional Review Board. IRB No.: 201700764B0.

\section{Disclosure}

The Authors declare that they have no conflict of interest regarding the publication of this article.

\section{Acknowledgements}

The Authors wish to thank the oncology nursing staff for providing the best possible care for the patients of this study.

\section{References}

1 Pannu HK and Oliphant M: The subperitoneal space and peritoneal cavity: basic concepts. Abdom Imaging 40: 27102722, 2015.

2 Edge SB, Byrd DR, Compton CC, Fritz AG, Greene FL, and Trotti A: Urinary bladder. In: AJCC Cancer Staging Manual. Seventh Edition. Edge SB, Byrd DR, Compton CC, Fritz AG, Greene FL and Trotti A (Eds.) Springer, New York, NY, pp. 497-505, 2010.

3 Sawant A, Bansal S and Pawar P: Early metastasis to anterior abdominal wall following radical cystectomy: A rare presentation. Urol Ann 8: 394-396, 2016.

4 Le O: Patterns of peritoneal spread of tumor in the abdomen and pelvis. World J Radio 5: 106-112, 2013.

5 Griffin N, Burke C and Grant LA: Common primary tumors of the abdomen and pelvis and their patterns of tumor spread as seen on multi-detector computed tomography. Insights Imaging 2: 205-214, 2011.

6 Shinagare AB, Ramaiya, NH, Jagannathan JP, Fennessy FM, Taplin ME and Van den Abbeele AD: Metastatic pattern of bladder cancer: correlation with the characteristics of the primary tumor. Am J Roentgenol 196: 117-122, 2011.

7 Turkbey B, BasaranC, Karcaaltincaba M, Akpinar E, Oguz B, Akata D, Ozmen MN and Akhan O: Peritoneal carcinomatosis in urinary bladder cancer. Clin Imaging 32: 192-195, 2008.

8 Chrysikopoulos $\mathrm{H}$, Maniatis V, Roussakis A, Pappas $\mathrm{J}$ and Andreou J: Peritoneal metastases from transitional cell carcinoma of the urinary tract: CT and MR imaging. Abdom Imaging 23: 91-95, 1998.

9 KundraV and Silverman PM: Imaging in oncology from the University of Texas M.D. Anderson Cancer Center. Imaging in the diagnosis, staging, and follow-up of cancer of the urinary bladder. Am J Roentgenol 180: 1045-1054, 2003

10 Black PC, Dinney CP, Brown GA, Kassouf W, Siefker-Radtke AO, Munsell MF, Grossman HB and Kamat AM: the role of radical cystectomy in patients with clinical $\mathrm{T} 4 \mathrm{~b}$ bladder cancer. Urol Oncol 29: 157-161, 2012.

11 Liberman D, Alasker A, Sun M, Ismail S, Lughezzani G, Jeldres C, Budaus L, Thuret R, Shariat SF, Widmer H, Perrotte P, Graefen M, Montorsi F and Karakiewicz PI: Radical cystectomy for patients with pT4 urothelial carcinoma in a large populationbased study. BJU Int 107: 905-911, 2011.
12 Eisenhauer EA, Therasse P, Bogaerts J, Schwartz LH, Sargent D, Ford R, Dancey J, Arbuck S, Gwyther S, Mooney M, Rubinstein L, Shankar L, Dodd L, Kaplan R, Lacombe D and Verweij J: New response evaluation criteria in solid tumors: revised RECIST guideline (version 1.1). Eur J Cancer 45: 228247, 2009.

13 Bessell EM, Price, HM, McMillan, PJL: The measurement of the regression of carcinoma of the bladder using ultrasonography and CT scanning during and after radical radiotherapy. Radiother Oncol 19: 145-157, 1990.

14 Hadjiiski L, Weizer AZ, Alva A, Caoili EM, Cohan RH, Cha K and, Chan HP: Treatment response assessment for bladder cancer on CT based on computerized volume analysis, WHO and RECIST Criteria. Am J Roentgenol 205: 348-352, 2015.

15 Lee SJ, Lee EJ, Kim SK, Jeong P, Cho YH, Yun SJ, Kim S, Kim GY, Choi YH, Cha EJ, Kim WJ and Moon SK: Identification of pro-inflammatory cytokines associated with muscle invasive bladder cancer: the roles of IL-5, IL-20. and IL-28A. PLoS One 7: e40267, 2012.

16 Liaw CC, Chang H, Liao TY, Wen MS, Yu CT and Juan,YH: Role of pulmonary veins in cancer progression from a computed tomography viewpoint. J Oncol 2016: 1872627, 2016.

17 Liao TY, Hsu HC, Wen MS, Juan YH, Hung YH and Liaw CC: Venous thrombosis mainly related to iliofemoral venous obstruction by external tumor compression in cancer patients. Case Rep Oncol 9: 760-771, 2016.

18 Als AB, Sengelov L and Von Der Maase H: Gemcitabine and cisplatin in locally advanced and metastatic bladder cancer: 3or 4-week schedule? Acta Oncol 47: 110-119, 2008.

19 Lin CC, Hsu CH, Huang CY, Cheng AL, Chen J, Vogelzang NJ and $\mathrm{Pu}$ YS: Weekly cisplatin plus infusional high-dose 5fluorouracil and leucovorin (P-HDFL) for metastatic urothelial carcinoma: an effective regimen with low toxicity. Cancer 106: 1269-1275, 2006.

20 Jan AS, Dola DE, Lombardi K and Gupta S: Tolerability of gemcitabine plus cisplatin for treatment of urothelial cancer in the elderly population. Clin Genitourin Cancer 14: e257$263,2016$.

21 Guancial EA, Roussel B, Bergsma DP and Bylund KC, Sahasrabudhe D, Messing E, Mohile SG and Fung C: Bladder cancer in the elderly patient: challenges and solutions. Clin Interv Aging 10: 939-949, 2015

22 G Zhang P, Zhang XD, Wang Y and Wang W: Feasibility of preand postoperative gemcitabine-plus-cisplatin systemic chemotherapy for the treatment of locally advanced urothelial carcinoma in kidney transplant patients. Transplant Proc 45: 3293-3297, 2013.

23 Mitry E, Taïeb J, Artru P, Boige V, Vaillant JN, Clavero-Fabri MC, Ducreux M and Rougier P: Combination of folinic acid, 5fluorouracil bolus and infusion, and cisplatin (LV5FU2-P regimen) in patients with advanced gastric or gastroesophageal junction carcinoma. Ann Oncol 15: 765-769, 2004.

24 Kasi PM and Grothey A: Chemotherapy maintenance. Cancer J 22: 199-204, 2016.

25 Esin E and Yalcin S: Maintenance strategy in metastatic colorectal cancer: a systematic review. Cancer Treat Rev 42: 8290, 2016.

26 Kalogirou C, Svistunov A, Krebs M, Lausenmeyer EM, Vergho $\mathrm{D}$, Riedmiller $\mathrm{H}$ and Kocot A: Maintenance monotherapy with gemcitabine following cisplatin-based primary combination 
chemotherapy in surgically treated advanced urothelial carcinoma: a matched-pair single institution analysis. Mol Clin Oncol 4: 636-642, 2016.

27 Yin M, Joshi M, Meijer RP and Glantz M: Neoadjuvant chemotherapy for muscle-invasive bladder cancer: a systematic review and two-step meta-analysis. Oncologist 21: 708-715, 2016.

28 Mossanen M, Lee F, Cheng H, Harris W, Shenoi J, Zhao S, Wang J, Champion T, Izard J, Gore JL, Porter MP, Yu EY and Wright JL: Nonresponse to neoadjuvant chemotherapy for muscle-invasive urothelial cell carcinoma of the bladder. Clin Genitourin Cancer 12: 210-213, 2014.

29 Sternberg CN, Skoneczna I, Kerst JM, Albers P, Fossa SD, Agerbaek M, Dumez H, de Santis M, Théodore C, Leahy MG, Chester JD, Verbaeys A, Daugaard G, Wood L, Witjes JA, de Wit R, Geoffrois L, Sengelov L, Thalmann G, Charpentier D, Rolland F, Mignot L, Sundar S, Symonds P, Graham J, Joly F, Marreaud S, Collette L and Sylvester R: Immediate versus deferred chemotherapy after radical cystectomy in patients with pT3-pT4 or N+ M0 urothelial carcinoma of the bladder (EORTC 30994): an intergroup, open-label, randomised phase 3 trial. Lancet Oncol 16: 76-86, 2015.

30 Tewari KS, Java JJ, Eskander RN, Monk BJ and Burger RA: Early initiation of chemotherapy following complete resection of advanced ovarian cancer associated with improved survival: NRG Oncology/Gynecologic Oncology Group study. Ann Oncol 27: 114-121, 2016.

31 Chen WC, Liaw CC, Chuang CK. Chen MF, Chen CS, Lin PY, Chang PL, Chu SH, Wu CT, and Hong JH: Concurrent cisplatin, 5-fluorouracil, leucovorin, and radiotherapy for invasive bladder cancer. Int J Radiat Oncol Biol Phys 56: 726-733, 2003.

32 Tao L, Qiu J, Jiang M, Song W, Yeh S, Yu H, Zang L, Xia S and Chang C: :Infiltrating T-cells promote bladder cancer progression via increasing IL1 $\rightarrow$ androgen receptor $\rightarrow \mathrm{HIF} 1 \alpha \rightarrow$ VEGFa signals. Mol Cancer Ther 15: 1943-1951, 2016.

33 Wang M, Zhao J, Zhang L, Wei F, Lian Y, Wu Y, Gong Z, Zhang S, Zhou J, Cao K, Li X, Xiong W, Li G, Zeng Z and Guo C: Role of tumor microenvironment in tumorigenesis. J Cancer 8 : 761-773, 2017.
34 Fearon KC, Voss AC and Hustead DS:Cancer Cachexia Study Group. Definition of cancer cachexia: effect of weight loss, reduced food intake, and systemic inflammation on functional status and prognosis. Am J Clin Nutr 83: 1345-1350, 2006.

35 Falang M, Marchetti A and Vignoli A: Coagulation and cancer: biological and clinical aspects. Thromb Haemost 1: 223-233, 2013.

36 Chang JC and Gross HM: Neoplastic fever responds to the treatment of an adequate dose of naproxen. J Clin Oncol 3: 552558,1985 .

37 Martin M, Wei H and Lu T: Targeting microenvironment in cancer therapeutics. Oncotarget 7: 52575-52583, 2016.

38 Kalluri R:The biology and function of fibroblasts in cancer. Nat Rev Cancer 16: 582-598, 2016.

39 Kharaishvili G, Simkova D, Bouchalova K, Gachechiladze M, Narsia $\mathrm{N}$ and Bouchal $\mathrm{J}$ : The role of cancer-associated fibroblasts, solid stress and other microenvironmental factors in tumor progression and therapy resistance. Cancer Cell Int 14: 41, 2014.

40 Sandoval P, Jiménez-Heffernan JA and Rynne-Vidal Á: Carcinoma-associated fibroblasts derive from mesothelial cells via mesothelial-to-mesenchymal transition in peritoneal metastasis. J Pathol 231: 517-531, 2013.

41 Midwood KS, Williams, LV and Schwarzbauer JE:Tissue repair and the dynamics of the extracellular matrix. Int J Biochem Cell Biol 36: 1031-1037, 2004.
Received August 4, 2017

Revised August 23, 2017

Accepted August 25, 2017 